\title{
Twisted higher spin Dirac operators
}

\author{
H. De Schepper, D. Eelbode and T. Raeymaekers
}

\begin{abstract}
In this paper, we define twisted higher spin Dirac operators and explain how these invariant differential operators can be used to define more general higher spin Dirac operators acting on functions $f(\underline{x})$ on $\mathbb{R}^{m}$ which then take values in general half-integer representations for the spin group.
\end{abstract}

Mathematics Subject Classification (2010). 30G35, 42 B35.

Keywords. Invariant operators, twisted Dirac operator, higher spin analysis.

\section{Introduction}

Classical Clifford analysis is usually defined as a function theory generalizing complex analysis to the case of arbitrary dimension $m \in \mathbb{Z}$ (considered as a formal parameter). Note that the case $m \leq 0$ corresponds to super Clifford analysis, see e.g. [7] and the references mentioned therein. At the same time, Clifford analysis is a refinement of classical harmonic analysis in $\mathbb{R}^{m}$. The main operator of interest, lying at the very heart of this function theory, is the so-called Dirac operator. This is a conformally invariant first order differential operator, acting on spinor-valued functions. Note that one often focusses on the rotational invariance with respect to the spin group or its orthogonal Lie algebra $\mathfrak{s o}(m)$. We refer the interested reader to the standard references $[1,8,13]$, in which this Dirac operator is studied from a function theoretical point of view (i.e. studying polynomial null-solutions, integral representations, special functions, etcetera).

Within the general theory of Riemannian spin manifolds, there exists an entire system of conformally invariant elliptic first order differential operators $\mathcal{D}$, see $[3,12,17,18]$. In recent years, Clifford analysis has shown to offer an elegant framework to study the aforementioned function theoretical problems not only for the Dirac operator, but also for far-reaching generalizations of it, acting on functions which take their values in arbitrary half-integer irreducible spin-representations. The earliest generalizations involved the so-called Rarita-Schwinger operator (shortly: the RS-operator), 
again inspired by equations coming from theoretical physics. In e.g. $[4,5]$ one can find the function theoretical fundaments for spin-invariant operators acting on functions taking values in irreducible modules of $\mathfrak{s o}(m)$ with highest weight $\left(l_{1}+\frac{1}{2}, \frac{1}{2}, \cdots, \frac{1}{2}\right)$, where $l_{1} \geq 1$.

The present paper is part of a program to study general spin-invariant operators and their polynomial solutions, in which both the dimension and the highest weight of the space of values are treated as parameters. In a recent paper [9] we have established explicit definitions for these operators, which we will refer to as higher spin Dirac operators (or HSD-operators for short), as operators acting on spinor-valued functions in several variables. A special case is the operator $\mathcal{Q}_{l_{1}, l_{2}}$, constructed and studied in [2], which acts on polynomials taking values in irreducible $\mathfrak{s o}(m)$-representations with highest weight $\left(l_{1}+\frac{1}{2}, l_{2}+\frac{1}{2}, \frac{1}{2} \cdots, \frac{1}{2}\right)$, with $l_{1} \geq l_{2} \geq 1$.

Rotationally invariant differential operators are usually constructed using the Stein-Weiss method [18]: this means that one acts with the gradient operator $\nabla$ on functions $f(x)$ taking values in the desired representation $\mathbb{V}$ for the orthogonal Lie algebra (adding a suitable conformal weight in case the resulting operators are meant to be conformally invariant). In view of the fact that $\nabla f(x)$ transforms as an element of $\mathbb{C}^{m} \otimes \mathbb{V}$ under $\mathfrak{s o}(m)$, it then suffices to make a suitable projection on the irreducible summands appearing in the tensor product (roughly speaking: 'monogenics times vectors' in our case of interest) in order to find invariant operators. In these cases (i.e. for half-integer highest weight representations), there is an alternative: instead of acting with the gradient one can also act with the Dirac operator $\underline{\partial}_{x}$, hereby fully exploiting the power of the Clifford multiplication. From the point of view of invariant operators, the Dirac operator is (strictly speaking) only defined on spinor-valued functions (i.e. for $\mathbb{V}$ the spinor space), which is why this method is referred to as the 'twisted Dirac operator method'. The word 'twisted' hereby captures the idea that the Dirac operator acts on functions taking values in the 'wrong' space. One is then again led to a tensor product, which is however different from the one mentioned above (roughly speaking: 'harmonics times spinors'). In the present paper, we will show that HSD operators can also be constructed using twisted operators 'of a lower order' (to be clarified in what follows): instead of working with the gradient or Dirac operator, we thus choose yet another operator acting on functions with values in $\mathbb{V}$, hereby again reducing the construction of invariant operators to a tensor product. However, as we will see, this time the tensor product will only contain two relevant components (which is considerably less than the number of components obtained using e.g. the twisted Dirac operator). In a sense, this approach leads to an inductive pattern (which was already mentioned in [9], although there it was a purely formal observation, which we now elaborate from the representation theoretical point of view), which will in its own term be exploited when constructing null solutions (in an upcoming paper). 
The outline of this paper is as follows. In Section 2, we will give some general results and notations from Clifford analysis. This will turn out to be very useful for our purposes, since arbitrary irreducible half-integer representations for the spin group can be realized by means of polynomial models in the language of Clifford analysis. In Section 3, we will introduce the twisted Dirac operator and its use in higher spin analysis. In Section 4, the twisted RS-operators are defined and used to obtain an alternative realization for the operator $\mathcal{Q}_{l_{1}, l_{2}}$. We have chosen to include this operator as a special case, in order to illustrate the more general procedure from Section 5, in which the operators $\mathcal{Q}_{l_{1}, \ldots, l_{k}}$ are obtained through an inductive procedure involving twisted HSD-operators.

\section{Clifford analysis background}

Let $\mathbb{R}_{m}$ be the universal Clifford algebra generated by an orthonormal basis $\left(\underline{e}_{1}, \ldots, \underline{e}_{m}\right)$ for the $m$-dimensional vector space $\mathbb{R}^{m}$ and let $\mathbb{C}_{m}=\mathbb{R}_{m} \otimes \mathbb{C}$ be its complexification. The multiplication in these algebras is governed by the relations $\underline{e}_{i} \underline{e}_{j}+\underline{e}_{j} \underline{e}_{i}=-2 \delta_{i j}$ for all $i, j=1, \ldots, m$. Any vector $\underline{x}=$ $\left(x_{1}, \ldots, x_{m}\right) \in \mathbb{R}^{m}$ will be identified with the corresponding Clifford vector $\underline{x}=\sum_{j=1}^{m} x_{j} \underline{e}_{j}$. Inside the complex algebra $\mathbb{C}_{m}$, one can then realize the so-called spinor space $\mathbb{S}$ as a minimal left ideal, see e.g. [8] for the explicit definition. For odd dimensions $m$, the vector space $\mathbb{S}$ defines a model for the basic half-integer representation for the spin group described by the highest weight $\left(\frac{1}{2}, \frac{1}{2}, \cdots, \frac{1}{2}\right)$ under the action $\psi \mapsto s \psi$, for all $\psi \in \mathbb{S}$ and $s \in \operatorname{Spin}(m)$. Note that the spin group itself can be realized inside the Clifford algebra by means of

$$
\operatorname{Spin}(m)=\left\{s=\prod_{j=1}^{2 k} s_{j}: k \in \mathbb{N}, s_{j} \in S^{m-1}\right\},
$$

where $S^{m-1} \subset \mathbb{R}^{m}$ denotes the unit sphere in $\mathbb{R}^{m}$. In even dimensions $m$, the spinor space $\mathbb{S}=\mathbb{S}^{+} \oplus \mathbb{S}^{-}$is reducible and decomposes into a direct sum of positive and negative spinors. Both spaces are irreducible under the multiplicative action of $\operatorname{Spin}(m)$, with highest weights $\left(\frac{1}{2}, \frac{1}{2}, \cdots, \frac{1}{2}\right)$ and $\left(\frac{1}{2}, \frac{1}{2}, \cdots, \frac{1}{2},-\frac{1}{2}\right)$. Lying at the core of Clifford analysis is the unique conformally invariant elliptic differential operator acting between spinor-valued functions, known as the Dirac operator:

$$
\underline{\partial}_{x}=\sum_{j=1}^{m} \underline{e}_{j} \partial_{x_{j}} .
$$

This operator factorizes the Laplace operator $\Delta_{x}=-\underline{\partial}_{x}^{2}$, reflecting the statement that Clifford analysis refines harmonic analysis on $\mathbb{R}^{m}$. Note that in case of even dimensions $m$ the Dirac operator acts as a linear map $\underline{\partial}_{x}$ : $\mathcal{C}^{\infty}\left(\mathbb{R}^{m}, \mathbb{S}^{ \pm}\right) \rightarrow \mathcal{C}^{\infty}\left(\mathbb{R}^{m}, \mathbb{S}^{\mp}\right)$, which means that the action of this operator changes the parity of the spinors. For the sake of notational convenience, we will therefore restrict ourselves to the odd-dimensional case in this article. 
Note however that the even-dimensional case is not conceptually different. One only needs to take into account that also the HSD-operators will change the parity, and can therefore be expressed in terms of a skew-symmetric matrix.

A crucial piece of information is the fact that not only the spinor space $\mathbb{S}$, but also other irreducible half-integer $\operatorname{Spin}(m)$-representations can be characterized in the language of Clifford algebras and Clifford analysis, see e.g. [6]. This is done using the notion of Clifford analysis in several vector variables $\underline{u}_{i} \in \mathbb{R}^{m}$. Throughout this paper, we will assume that the number of vector variables $k \leq\left\lfloor\frac{m}{2}\right\rfloor$, which means that we will restrict ourselves to the so-called stable range [14]. From now on, we will denote the corresponding Dirac operators $\underline{\partial}_{u_{i}}$ by $\underline{\partial}_{i}$ and reserve the underlined letter $\underline{u}$ for 'dummy variables'. The notation $\langle\cdot, \cdot\rangle$ is reserved vor the Euclidean inner product on $\mathbb{R}^{m}$, which means that e.g. $\left\langle\underline{\partial}_{x}, \underline{\partial}_{y}\right\rangle=\sum_{j=1}^{m} \partial_{x_{j}} \partial_{y_{j}}$. In order to define our models for these spin-representations, we need particular classes of polynomials which are introduced in the following definitions.

Definition 1. A function $f: \mathbb{R}^{k m} \rightarrow \mathbb{C}:\left(\underline{u}_{1}, \ldots, \underline{u}_{k}\right) \mapsto f\left(\underline{u}_{1}, \ldots, \underline{u}_{k}\right)$ is called simplicial harmonic if it satisfies the system

$$
\begin{aligned}
& \left\langle\underline{\partial}_{i}, \underline{\partial}_{j}\right\rangle f=0, \text { for all } i, j=1, \ldots, k \\
& \left\langle\underline{u}_{i}, \underline{\partial}_{j}\right\rangle f=0, \text { for all } 1 \leq i<j \leq k .
\end{aligned}
$$

The vector space of $\mathbb{C}$-valued simplicial harmonic polynomials which are homogeneous of degree $l_{i}$ in $\underline{u}_{i}$ will be denoted by $\mathcal{H}_{l_{1}, \ldots, l_{k}}$, or $\mathcal{H}_{\lambda}$ for short (with $\left.\underline{\lambda}=\left(l_{1}, \ldots, l_{k}\right)\right)$ where, from now on, we assume that $l_{1} \geq \cdots \geq l_{k}$ (the dominant weight condition).

Definition 2. A function $f: \mathbb{R}^{k m} \rightarrow \mathbb{S}:\left(\underline{u}_{1}, \ldots, \underline{u}_{k}\right) \mapsto f\left(\underline{u}_{1}, \ldots, \underline{u}_{k}\right)$ is called simplicial monogenic if it satisfies the system

$$
\begin{aligned}
\underline{\partial}_{i} f & =0, \text { for all } i=1, \ldots, k \\
\left\langle\underline{u}_{i}, \underline{\partial}_{j}\right\rangle f & =0, \text { for all } 1 \leq i<j \leq k .
\end{aligned}
$$

The vector space of $\mathbb{S}$-valued simplicial monogenic polynomials which are homogeneous of degree $l_{i}$ in $\underline{u}_{i}$ will be denoted by $\mathcal{S}_{l_{1}, \ldots, l_{k}}$ or $\mathcal{S}_{\underline{\lambda}}$ for short $\left(\underline{\lambda}=\left(l_{1}, \ldots, l_{k}\right)\right)$, where again, from now on, we assume that $l_{1} \geq \cdots \geq l_{k}$. The following definition involves weaker conditions on the $\mathbb{S}$-valued functions, but will nevertheless be crucial in what follows.

Definition 3. A function $f: \mathbb{R}^{k m} \rightarrow \mathbb{S}:\left(\underline{u}_{1}, \ldots, \underline{u}_{k}\right) \mapsto f\left(\underline{u}_{1}, \ldots, \underline{u}_{k}\right)$ is called monogenic in $k$ variables if it satisfies $\underline{\partial}_{i} f=0$, for all $1 \leq i \leq k$.

The vector space of $\mathbb{S}$-valued monogenic polynomials which are $l_{i}$-homogeneous in $\underline{u}_{i}$ will be denoted by $\mathcal{M}_{l_{1}, \ldots, l_{k}}$ or $\mathcal{M}_{\underline{\lambda}}$ for short $\left(\underline{\lambda}=\left(l_{1}, \ldots, l_{k}\right)\right)$, again with $l_{1} \geq \cdots \geq l_{k}$. Each of these polynomial vector spaces can be seen as a module for the spin group under the induced regular representation. For $\mathbb{C}$-valued polynomials, this is the $H$-action

$$
H(s) P\left(\underline{u}_{1}, \ldots, \underline{u}_{k}\right):=P\left(\bar{s} \underline{u}_{1} s, \ldots, \bar{s} \underline{u}_{k} s\right), \quad s \in \operatorname{Spin}(m),
$$


whereas for $\mathbb{S}$-valued polynomials this becomes the $L$-action

$$
L(s) P\left(\underline{u}_{1}, \ldots, \underline{u}_{k}\right):=s P\left(\bar{s} \underline{u}_{1} s, \ldots, \bar{s} \underline{u}_{k} s\right), \quad s \in \operatorname{Spin}(m) .
$$

In e.g. [6, 13], it was proven that under this action, the $\operatorname{Spin}(m)$-modules $\mathcal{H}_{l_{1}, \cdots, l_{k}}$ define a model for the irreducible highest weight representation characterized by means of

$$
\mathcal{H}_{l_{1}, \cdots, l_{k}} \rightarrow\left(l_{1}, \cdots, l_{k}, 0, \cdots, 0\right)=:\left(l_{1}, \cdots, l_{k}\right),
$$

whereas the $\operatorname{Spin}(m)$-modules $\mathcal{S}_{l_{1}, \cdots, l_{k}}$ define a model for the irreducible highest weight representation characterized by means of

$$
\mathcal{S}_{l_{1}, \cdots, l_{k}} \rightarrow\left(l_{1}+\frac{1}{2}, \cdots, l_{k}+\frac{1}{2}, \frac{1}{2}, \cdots, \frac{1}{2}\right)=:\left(l_{1}, \cdots, l_{k}\right)^{\prime} .
$$

As shown in the notations above, we will omit redundant zeros in the highest weight, and denote the Cartan product with the spinor space $\mathbb{S}$ by means of a prime. Note that when $m=2 n$, a parity index needs to be added to the spaces of simplicial monogenics, according to the one for spinors.

Remark 1. In order for $\left(l_{1}, \ldots, l_{k}\right)$ of $\left(l_{1}, \ldots, l_{k}\right)^{\prime}$ to be a Spin $(m)$-representation, we need that $l_{1} \geq l_{2} \geq \cdots \geq l_{k}$, the so called dominant weight condition. That is the reason why we imposed this condition on the spaces $\mathcal{S}_{\underline{\lambda}}$ and $\mathcal{H}_{\underline{\lambda}}$.

On functions $f\left(\underline{x} ; \underline{u}_{1}, \cdots, \underline{u}_{k}\right)$ taking values in the vector space $\mathcal{S}_{l_{1}, \ldots, l_{k}}$, we can define operators playing the role of $\underline{\partial}_{x}$ for $\mathbb{S}$-valued functions (the HSD-operators mentioned in the introduction). These are first order $\operatorname{Spin}(m)$ invariant differential operators $\mathcal{Q}_{\underline{\lambda}}$, with $\underline{\lambda}=\left(l_{1}, \cdots, l_{k}\right)$, uniquely defined up to a multiplicative constant (they are even conformally invariant, but this fact will not be needed in the present paper):

$$
\mathcal{Q}_{\underline{\lambda}}: \mathcal{C}^{\infty}\left(\mathbb{R}^{m}, \mathcal{S}_{\underline{\lambda}}\right) \rightarrow \mathcal{C}^{\infty}\left(\mathbb{R}^{m}, \mathcal{S}_{\underline{\lambda}}\right): f\left(\underline{x} ; \underline{u}_{1}, \ldots, \underline{u}_{k}\right) \mapsto \mathcal{Q}_{\underline{\lambda}} f\left(\underline{x} ; \underline{u}_{1}, \ldots, \underline{u}_{k}\right) .
$$

The existence and uniqueness of such (conformally) invariant differential operators follows from e.g. Fegan's result [12]; their explicit form as an operator acting on spinor-valued functions in several vector variables was determined in $[9]$ as

$$
\underline{\mathcal{Q}}_{\underline{\lambda}}=\left(\prod_{p=1}^{k}\left(1+\frac{\underline{u}_{p} \underline{\partial}_{p}}{m+2 l_{p}-2 p}\right)\right) \underline{\partial}_{x} .
$$

Remark 2. Note that we have to see this product as an ordered product, because elements in $\mathbb{C}_{m}$ do not necessarily commute. The product above is taken in the ascending order, from left to right.

In case $k=1$, we obtain the well-known Rarita-Schwinger operator (see e.g. [4]):

$$
\mathcal{Q}_{l_{1}}=\left(1+\frac{\underline{u}_{1} \underline{\partial}_{1}}{m+2 l_{1}-2}\right) \underline{\partial}_{x}
$$

The case $k=2$ has been extensively studied as well, see e.g. [11]:

$$
\mathcal{Q}_{l_{1}, l_{2}}=\left(1+\frac{\underline{u}_{1} \underline{\partial}_{1}}{m+2 l_{1}-2}\right)\left(1+\frac{\underline{u}_{2} \underline{\partial}_{2}}{m+2 l_{2}-4}\right) \underline{\partial}_{x} .
$$


In the next section, we will develop an alternative approach to construct these operators inductively, using the notion of twisted higher spin operators.

\section{The twisted Dirac operator}

In this section we consider the twisted Dirac operator, which can be defined on $\mathcal{H}_{\underline{\lambda}}$-valued functions (for arbitrary $\underline{\lambda}$ ) and enables us to define the aforementioned HSD-operators. More explicitly, we have the following.

Definition 4. For arbitrary integer-valued highest weights $\underline{\lambda}$ for Spin $(m)$, the twisted Dirac operator on $\left(\mathcal{H}_{\underline{\lambda}} \otimes \mathbb{S}\right)$-valued polynomials is defined by means of

$$
\underline{\partial}_{x}^{T}:=\mathbf{1}_{\underline{\lambda}} \otimes \underline{\partial}_{x}: \mathcal{C}^{\infty}\left(\mathbb{R}^{m}, \mathcal{H}_{\underline{\lambda}} \otimes \mathbb{S}\right) \rightarrow \mathcal{C}^{\infty}\left(\mathbb{R}^{m}, \mathcal{H}_{\underline{\lambda}} \otimes \mathbb{S}\right)
$$

In order to explain why this operator is so useful to define HSD-operators acting on $\mathcal{S}_{\underline{\underline{\lambda}}}$-valued polynomials, we invoke the following result.

Lemma 1 (e.g. [10]). As a Spin $(m)$-representation, the tensor product $\mathcal{H}_{\underline{\lambda}} \otimes \mathbb{S}$ can be decomposed as the direct sum of (at most) $2^{|\underline{\lambda}|}$ irreducible Spin(m)modules, each one appearing with multiplicity 1 :

$$
\mathcal{H}_{\underline{\lambda}} \otimes \mathbb{S} \cong \bigoplus_{i_{1}=0}^{1} \cdots \bigoplus_{i_{k}=0}^{1}\left(l_{1}-i_{1}, \ldots, l_{k}-i_{k}\right)^{\prime}
$$

Each summand $\left(l_{1}-i_{1}, \ldots l_{k}-i_{k}\right)^{\prime}$ is contained in the decomposition as long as its highest weight satisfies the dominant weight condition.

Note that it follows from this lemma that $\mathcal{S}_{\underline{\lambda}}$ is a submodule of $\mathcal{H}_{\underline{\lambda}} \otimes \mathbb{S}$, which is precisely what lies behind Definition 4 . Note also that all other modules are isomorphically embedded into the tensor product, but we will omit the embedding factor (unless explicitly mentioned). Using the method of constructing conformally invariant operators by means of generalized gradients (see e.g. $[12,18]$ ), one can deduce from the lemma above that the twisted Dirac operator $\mathbf{1}_{\underline{\lambda}} \otimes \underline{\partial}_{x}$ can be written as the sum of at most $|\underline{\lambda}|+1$ first order differential operators: a HSD-operator $\mathcal{Q}_{\underline{\lambda}}$ and (at most) $|\underline{\lambda}|$ twistor operators $\mathcal{T}_{\underline{\lambda}-\delta_{i}}$, defined as the unique first order differential operators mapping $\mathcal{S}_{\underline{\lambda}}$-valued functions to $\mathcal{S}_{\underline{\lambda}-\delta_{i}}$-valued functions. Each of these operators 
is defined through the following scheme:

$$
\mathcal{C}^{\infty}\left(\mathbb{R}^{m}, \mathcal{S}_{\underline{\lambda}}\right) \underline{\partial}_{x}^{T}>\mathcal{C}^{\infty}\left(\mathbb{R}^{m}, \mathcal{H}_{\underline{\lambda}} \otimes \mathbb{S}\right)
$$

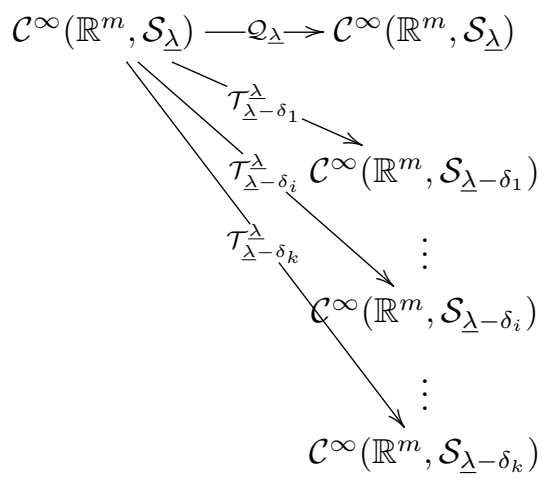

Here, $\underline{\lambda}-\delta_{i}$ stands for $\left(l_{1}, \ldots, l_{i}-1, \ldots, l_{k}\right)$. Note that there are less twistor operators than summands in the decomposition from Lemma 1, which follows from Fegan's result on existence or easy Clifford analysis calculations.

Before we turn our attention to arbitrary twisted HSD-operators, we will illustrate our approach by means of a low order example: for $k=1$ we encounter the classical Dirac operator, leading by means of its decomposition to the definition of the RS-operator. Here, Lemma 1 reduces to the classical Fischer decomposition (this time including the embedding factor):

$$
\mathcal{H}_{l_{1}} \otimes \mathbb{S}=\mathcal{S}_{l_{1}} \oplus \underline{u}_{1} \mathcal{S}_{l_{1}-1} .
$$

Since

$$
f(\underline{x} ; \underline{u}) \in \mathcal{C}^{\infty}\left(\mathbb{R}^{m}, \mathcal{S}_{l_{1}}\right) \stackrel{\underline{\partial}_{x}^{T}}{\longrightarrow} \underline{\partial}_{x}^{T} f(\underline{x} ; \underline{u}) \in \mathcal{C}^{\infty}\left(\mathbb{R}^{m}, \mathcal{H}_{l_{1}} \otimes \mathbb{S}\right),
$$

we only need to project $\underline{\partial}_{x}^{T} f$ onto the space $\mathcal{C}^{\infty}\left(\mathbb{R}^{m}, \mathcal{S}_{l_{1}}\right)$ in order to obtain an expression for the RS-operator $\mathcal{Q}_{l_{1}}$, in view of Lemma 1. Schematically, we have:

$$
\begin{gathered}
\mathcal{C}^{\infty}\left(\mathbb{R}^{m}, \mathcal{S}_{l_{1}}\right)-\underline{\partial}_{x}^{T} \rightarrow \mathcal{C}^{\infty}\left(\mathbb{R}^{m}, \mathcal{H}_{l_{1}} \otimes \mathbb{S}\right) \\
=\quad \\
\mathcal{C}^{\infty}\left(\mathbb{R}^{m}, \mathcal{S}_{l_{1}}\right)-\mathcal{Q}_{l_{1}} \rightarrow \mathcal{C}^{\infty}\left(\mathbb{R}^{m}, \mathcal{S}_{l_{1}}\right) \\
{\mathcal{\mathcal { T } _ { l _ { 1 } - 1 } ^ { l _ { 1 } }}}_{\mathcal{C}^{\infty}\left(\mathbb{R}^{m}, \mathcal{S}_{l_{1}-1}\right)}
\end{gathered}
$$

Here, we can see that the twisted Dirac operator decomposes into two operators. These are two natural invariant operators acting on the $\mathcal{S}_{l_{1}}$-valued functions under consideration: the Rarita-Schwinger operator $\mathcal{Q}_{l_{1}}$ and a twistor operator $\mathcal{T}_{l_{1}-1}^{l_{1}}$ (see e.g. [4]). 
Remark 3. As $k$ increases, it will be harder to obtain a projection operator on $\mathcal{S}_{\lambda}$, since the scheme (2) will contain more arrows.

Remark 4. When using $\underline{\partial}_{x}$ to construct $\mathcal{Q}_{l_{1}}$, one actually uses the natural operator acting on functions taking values in the space "with one dummy variable less" (here: spinors). This observation has inspired us to follow a similar approach for more general values.

As illustrated above, explicit realisations for the HSD-operators can be obtained by decomposing the twisted Dirac operator. However, our main aim is to eventually describe the polynomial null-solutions for general HSDoperators. As $k$ increases, the complexity of this kernel space increases as well (see e.g. [2]). This is why we will use a different approach to construct HSD-operators in the remainder of this article (using recursion), which will then lead to an alternative method to determine null-solutions. Instead of using the twisted Dirac operator, we will construct the HSD-operators using twisted HSD-operators of 'lower order'. This will be illustrated in the next section, where we will use the twisted RS-operator, in order to define the HSD-operator $\mathcal{Q}_{l_{1}, l_{2}}(k=2)$.

\section{The twisted Rarita-Schwinger operator}

The main aim of this section is to construct the HSD-operators $\mathcal{Q}_{l_{1}, l_{2}}$ using twisted RS-operators. The classical RS-operator is the HSD-operator of order 1 , defined on $\mathcal{S}_{l_{1}}$-valued functions $f\left(\underline{x} ; \underline{u}_{1}\right)$. It is however clear that the operator $\mathcal{Q}_{l_{1}}$, given by

$$
\mathbf{1}_{\mathbb{V}} \otimes \mathcal{Q}_{l_{1}}=\mathbf{1}_{\mathbb{V}} \otimes\left(1+\frac{\underline{u}_{1} \underline{\partial}_{1}}{m+2 l_{1}-2}\right) \underline{\partial}_{x},
$$

can act on any function space of the form $\mathcal{C}^{\infty}\left(\mathbb{R}^{m}, \mathbb{V} \otimes \mathcal{S}_{l_{1}}\right)$. Just as for the Dirac operator, this will then lead to the twisted RS-operator. In this paper, we will take $\mathbb{V}=\mathcal{H}_{l_{2}}$. The reason for this is that we eventually want to determine the expression for $\mathcal{Q}_{l_{1}, l_{2}}$ starting from the twisted RS-operator. This choice will prove to be very useful.

Definition 5. For any highest weight $\left(\mu_{1}, \ldots, \mu_{k-1}\right)$ with $l_{1} \geq \mu_{1}$, we define the twisted $R S$-operator by means of

$$
\begin{aligned}
\mathcal{Q}_{l_{1}}^{T}= & \mathbf{1}_{\left(\mu_{1}, \ldots, \mu_{k-1}\right)} \otimes \mathcal{Q}_{l_{1}}: \\
& \mathcal{C}^{\infty}\left(\mathbb{R}^{m}, \mathcal{H}_{\mu_{1}, \ldots, \mu_{k-1}} \otimes \mathcal{S}_{l_{1}}\right) \rightarrow \mathcal{C}^{\infty}\left(\mathbb{R}^{m}, \mathcal{H}_{\mu_{1}, \ldots, \mu_{k-1}} \otimes \mathcal{S}_{l_{1}}\right) .
\end{aligned}
$$

Note that we have chosen not to include the highest weight $\left(\mu_{1}, \ldots, \mu_{k-1}\right)$ in the symbol for the twisted $R S$-operator to avoid overloaded notations, although the precise definition obviously depends on the choice of these integers.

Remember that $\mathcal{Q}_{l_{1}, l_{2}}$ acts on functions taking values in $\mathcal{S}_{l_{1}, l_{2}}$. Recalling the meaning of this space as a vector space containing polynomial solutions to systems of differential equations, it is easily seen that the spin-module $\mathcal{S}_{l_{1}, l_{2}}$ is 
a subspace of $\mathcal{H}_{l_{2}} \otimes \mathcal{S}_{l_{1}}$. So we can determine $\mathcal{Q}_{l_{1}, l_{2}}$ by letting the twisted RSoperator act on functions in the space $\mathcal{C}^{\infty}\left(\mathbb{R}^{m}, \mathcal{S}_{l_{1}, l_{2}}\right)$ and projecting the result on the very same space afterwards. To prove the uniqueness of this projection, we need to prove that $\mathcal{S}_{l_{1}, l_{2}}$ is contained in $\mathcal{H}_{l_{2}} \otimes \mathcal{S}_{l_{1}}$ with multiplicity 1 . This is the subject of the following theorem, whose proof is rather technical and therefore postponed until Section 6.1.

Theorem 1. For each pair of integers $l_{1} \geq l_{2}>0$, we have that $\mathcal{S}_{l_{1}, l_{2}}$ and $\mathcal{S}_{l_{1}, l_{2}-1}$ are $\operatorname{Spin}(m)$-submodules of $\mathcal{H}_{l_{2}} \otimes \mathcal{S}_{l_{1}}$ with multiplicity 1 .

Let us then start from an arbitrary function $f\left(\underline{x} ; \underline{u}_{1}, \underline{u}_{2}\right) \in \mathcal{C}^{\infty}\left(\mathbb{R}, \mathcal{S}_{l_{1}, l_{2}}\right)$. After applying the twisted RS-operator (4), we obviously get that

$$
\mathcal{Q}_{l_{1}}^{T} f=\left(1+\frac{\underline{u}_{1} \underline{\partial}_{1}}{m+2 l_{1}-2}\right) \underline{\partial}_{x} f \in \operatorname{ker}\left(\underline{\partial}_{1}\right) .
$$

It is no longer true that $\mathcal{Q}_{l_{1}}^{T} f \in \operatorname{ker}\left(\underline{\partial}_{2},\left\langle\underline{u}_{1}, \underline{\partial}_{2}\right\rangle\right)$ but we do have that

$$
\mathcal{Q}_{l_{1}}^{T} f \in \operatorname{ker}\left(\underline{\partial}_{1}, \Delta_{2},\left\langle\underline{u}_{1}, \underline{\partial}_{2}\right\rangle^{2}\right),
$$

where $\Delta_{2}$ stands for the Laplace operator in the variable $\underline{u}_{2}$. This can easily be seen, due to the fact that $\mathcal{Q}_{l_{1}}^{T}$ projects on the kernel of $\underline{\partial}_{1}, \Delta_{2}$ commutes with $\mathcal{Q}_{l_{1}}^{T}$ and

$$
\left\langle\underline{u}_{1}, \underline{\partial}_{2}\right\rangle \mathcal{Q}_{l_{1}}^{T} f=-\frac{\underline{u}_{1} \underline{\partial}_{2}}{m+2 l_{1}-2} \underline{\partial}_{x} f .
$$

Since we know that the result is harmonic in the variable $\underline{u}_{2}$, we can use the monogenic decomposition (3):

$$
\mathcal{Q}_{l_{1}}^{T} f=F_{l_{2}}+\underline{u}_{2} F_{l_{2}-1},
$$

where $F_{l_{2}}$ and $F_{l_{2}-1}$ are both monogenic in $\underline{u}_{2}$. Applying $\underline{\partial}_{2}$ on both sides of the equation, explicit calculations involving the explicit expression for $\mathcal{Q}_{l_{1}}$ lead to

$$
\begin{aligned}
F_{l_{2}-1}=-\frac{\underline{\partial}_{2} \mathcal{Q}_{l_{1}}^{T} f}{m+2 l_{2}-2} & =-\frac{\left(m+2 l_{1}-2\right) \underline{\partial}_{2}-2\left\langle\underline{u}_{1}, \underline{\partial}_{2}\right\rangle \underline{\partial}_{1}}{\left(2 l_{1}+m-2\right)\left(2 l_{2}+m-2\right)} \underline{\partial}_{x} f \\
& =2 \frac{2 l_{1}+m}{\left(2 l_{1}+m-2\right)\left(2 l_{2}+m-2\right)}\left\langle\underline{\partial}_{2}, \underline{\partial}_{x}\right\rangle f .
\end{aligned}
$$

Notice that the operator $\left\langle\underline{\partial}_{2}, \underline{\partial}_{x}\right\rangle$ appearing here is (up to a multiplicative constant) the twistor operator $\mathcal{T}_{l_{1}, l_{2}-1}^{l_{1}, l_{2}}$, which is the unique first order differential operator acting between the following spaces:

$$
\left\langle\underline{\partial}_{2}, \underline{\partial}_{x}\right\rangle: \mathcal{C}^{\infty}\left(\mathbb{R}^{m}, \mathcal{S}_{l_{1}, l_{2}}\right) \rightarrow \mathcal{C}^{\infty}\left(\mathbb{R}^{m}, \mathcal{S}_{l_{1}, l_{2}-1}\right) .
$$

Remember that this operator also appeared in the scheme 2 (for $k=2$ ).

Defining $\pi_{l_{1}}\left[\underline{u}_{2}\right]$ as the projection of the multiplication operator $\underline{u}_{2}$ on the kernel of the Dirac operator $\underline{\partial}_{1}$, gives rise to a mapping

$$
\pi_{l_{1}}\left[\underline{u}_{2}\right]:=\left(1+\frac{\underline{u}_{1} \underline{\partial}_{1}}{m+2 l_{1}-2}\right) \underline{u}_{2}: \mathcal{C}^{\infty}\left(\mathbb{R}^{m}, \mathcal{S}_{l_{1}, l_{2}-1}\right) \rightarrow \mathcal{C}^{\infty}\left(\mathbb{R}^{m}, \mathcal{H}_{l_{2}} \otimes \mathcal{S}_{l_{1}}\right)
$$

we can prove the following proposition. 
Proposition 1. For all integers $l_{1} \geq l_{2}>0$ and for all $f\left(\underline{x} ; \underline{u}_{1}, \underline{u}_{2}\right) \in \mathcal{C}^{\infty}\left(\mathbb{R}^{m}\right.$, $\left.\mathcal{S}_{l_{1}, l_{2}}\right)$ with $\left\langle\underline{\partial}_{2}, \underline{\partial}_{x}\right\rangle f \neq 0$, there exists a unique constant $\gamma_{l_{2}} \in \mathbb{R}$ such that

$$
\mathcal{Q}_{l_{1}}^{T} f=\phi_{0}+\gamma_{l_{2}} \pi_{l_{1}}\left[\underline{u}_{2}\right]\left\langle\underline{\partial}_{2}, \underline{\partial}_{x}\right\rangle f
$$

with $\phi_{0}$ satisfying

$$
\underline{\partial}_{1} \phi_{0}=\underline{\partial}_{2} \phi_{0}=\left\langle\underline{u}_{1}, \underline{\partial}_{2}\right\rangle \phi_{0}=0 \text {. }
$$

This clearly means that $\phi_{0}=\mathcal{Q}_{l_{1}, l_{2}} f$. This constant is given by

$$
\gamma_{l_{2}}=\frac{2}{2 l_{2}+m-4} \text {. }
$$

Proof. Let us consider a function $f \in \mathcal{C}^{\infty}\left(\mathbb{R}^{m}, \mathcal{S}_{l_{1}, l_{2}}\right)$ with $\left\langle\underline{\partial}_{2}, \underline{\partial}_{x}\right\rangle f \neq 0$, and define $\phi_{0}$ by means of

$$
\phi_{0}:=\mathcal{Q}_{l_{1}}^{T} f-\gamma_{l_{2}} \pi_{l_{1}}\left[\underline{u}_{2}\right]\left\langle\underline{\partial}_{2}, \underline{\partial}_{x}\right\rangle f \in \mathcal{C}^{\infty}\left(\mathbb{R}^{m}, \mathcal{H}_{l_{2}} \otimes \mathcal{S}_{l_{1}}\right),
$$

where the constant $\gamma_{2}$ is to be fixed in such a way that $\phi_{0}$ indeed satisfies the requirements mentioned above. In view of the fact that $\mathcal{Q}_{l_{1}}^{T} f \in \operatorname{ker} \Delta_{2}$, we can use (3) to arrive at

$$
\mathcal{Q}_{l_{1}}^{T} f=F_{l_{2}}+\underline{u}_{2} F_{l_{2}-1}
$$

where both functions $F_{i}$ are homogeneous of degree $i$ and monogenic in $\underline{u}_{2}$. Applying the Dirac operator $\underline{\partial}_{2}$ on both expressions for $\mathcal{Q}_{l_{1}}^{T} f$ gives us:

$$
-\left(2 l_{2}+m-2\right) F_{l_{2}-1}=\underline{\partial}_{2} \phi_{0}-\gamma_{l_{2}} \frac{\left(2 l_{1}+m\right)\left(2 l_{2}+m-2\right)}{2 l_{1}+m-2}\left\langle\underline{\partial}_{2}, \underline{\partial}_{x}\right\rangle f .
$$

Using equation (5), we thus get that

$$
\underline{\partial}_{2} \phi_{0}=\left(\gamma_{l_{2}} \frac{\left(2 l_{1}+m\right)\left(2 l_{2}+m-2\right)}{2 l_{1}+m-2}-\frac{2\left(2 l_{1}+m\right)}{2 l_{1}+m-2}\right)\left\langle\underline{\partial}_{2}, \underline{\partial}_{x}\right\rangle f .
$$

If we choose

$$
\gamma_{l_{2}}=\frac{2}{2 l_{2}+m-4}
$$

one immediately sees that the proposition holds.

Remark 5. If $f \in \mathcal{C}^{\infty}\left(\mathbb{R}^{m}, \mathcal{S}_{l_{1}, l_{2}}\right) \cap \operatorname{ker}\left\langle\underline{\partial}_{2}, \underline{\partial}_{x}\right\rangle$, the proposition above reduces to

$$
\mathcal{Q}_{l_{1}}^{T} f \in \mathcal{C}^{\infty}\left(\mathbb{R}^{m}, \mathcal{S}_{l_{1}, l_{2}}\right) .
$$

Note that the operator $\gamma_{l_{2}} \pi_{l_{1}}\left[\underline{u}_{2}\right]\left\langle\underline{\partial}_{2}, \underline{\partial}_{x}\right\rangle$ is nothing but the operator $\mathcal{T}_{l_{1}, l_{2}-1}^{l_{1}, l_{2}}=\left\langle\underline{\partial}_{2}, \underline{\partial}_{x}\right\rangle$ and an embedding factor $\gamma_{l_{2}} \pi_{l_{1}}\left[\underline{u}_{2}\right]$, which means that we get the following scheme for the action of $\mathcal{Q}_{l_{1}}^{T}$ (up to isomorphic embeddings):

$$
\begin{array}{r}
\mathcal{C}^{\infty}\left(\mathbb{R}^{m}, \mathcal{S}_{l_{1}, l_{2}}\right)-\mathcal{Q}_{l_{1}}^{T}>\mathcal{C}^{\infty}\left(\mathbb{R}^{m}, \mathcal{H}_{l_{2}} \otimes \mathcal{S}_{l_{1}}\right) \\
\mathcal{C}^{\infty}\left(\mathbb{R}^{m}, \mathcal{S}_{l_{1}, l_{2}}\right)-\mathcal{Q}_{l_{1}, l_{2}}>\mathcal{C}^{\infty}\left(\mathbb{R}^{m}, \mathcal{S}_{l_{1}, l_{2}}\right) \\
\mathcal{T}_{l_{1}, l_{2}-1}^{l_{1}, l_{2}} \\
\mathcal{C}^{\infty}\left(\mathbb{R}^{m}, \mathcal{S}_{l_{1}, l_{2}-1}\right)
\end{array}
$$


Due to Theorem 1, this decomposition is unique.

\section{The twisted higher spin Dirac operator}

Similar to the derivation of $\mathcal{Q}_{l_{1}, l_{2}}$ in the previous section, we can now obtain an explicit realization for the most general HSD-operators, using a related twisted HSD-operator of 'lower order'. Suppose $\underline{\lambda}=\left(l_{1}, \ldots, l_{k}\right)$, with $l_{1} \geq$ $\cdots \geq l_{k}>0$. The standard HSD-operator in $k$ vector variables was defined as the first order differential operator:

$$
\mathcal{Q}_{\underline{\lambda}}: \mathcal{C}^{\infty}\left(\mathbb{R}^{m}, \mathcal{S}_{\underline{\lambda}}\right) \rightarrow \mathcal{C}^{\infty}\left(\mathbb{R}^{m}, \mathcal{S}_{\underline{\lambda}}\right) .
$$

Just as for the twisted RS-operator, we introduce the following definition.

Definition 6. The twisted HSD-operator $\mathcal{Q}_{\underline{\lambda}}^{T}$ is the operator

$$
\mathcal{Q}_{\underline{\lambda}}^{T}=\mathbf{1}_{\mathbb{V}} \otimes \mathcal{Q}_{\underline{\lambda}}: \mathcal{C}^{\infty}\left(\mathbb{R}^{m}, \mathbb{V} \otimes \mathcal{S}_{\underline{\lambda}}\right) \rightarrow \mathcal{C}^{\infty}\left(\mathbb{R}^{m}, \mathbb{V} \otimes \mathcal{S}_{\underline{\lambda}}\right)
$$

acting on $\mathbb{V}$-valued simplicial monogenics. Note that we again prefer not to mention this space $\mathbb{V}$ explicitly in the symbol for the twisted HSD-operator, in order to avoid overloaded notations.

Once again, note that the difference between the ordinary HSD-operator and its twisted version lies in the values of the functions $f(\underline{x})$ these operators are meant to act on. The 'twisted' refers to the fact that this operator acts on a 'bigger' space than the canonical domain of the ordinary HSD-operator. Now, let $\underline{\lambda}^{+}=\left(l_{1}, \ldots, l_{k}, l_{k+1}\right)$ be a dominant highest weight. In order to use this twisted HSD-operator to construct more complicated HSD-operators, one must choose $\mathbb{V}$ in such a way that $\mathcal{S}_{\underline{\lambda}^{+}} \subset \mathbb{V} \otimes \mathcal{S}_{\underline{\lambda}}$. We will prove that $\mathbb{V}=\mathcal{H}_{l_{k+1}}$, with $l_{k} \geq l_{k+1}>0$, fits this purpose.

In view of our polynomial models, it is easily seen that $\mathcal{S}_{\underline{\lambda}^{+}}$indeed is a subspace of the tensor product $\mathcal{H}_{l_{k+1}} \otimes \mathcal{S}_{\underline{\lambda}}$. In order to obtain a HSDoperator $\mathcal{Q}_{\lambda^{+}}$which is well-defined, we need to prove that $\mathcal{S}_{\underline{\lambda}^{+}}$is contained in this tensor product with multiplicity one.

Theorem 2. Defining the (dominant) highest weights

$$
\underline{\lambda}^{-}=\left(l_{1}, \ldots, l_{k}, l_{k+1}-1\right) \text { and } \underline{\lambda}^{+}=\left(l_{1}, \ldots, l_{k}, l_{k+1}\right),
$$

both $\mathcal{S}_{\underline{\lambda}^{-}}$and $\mathcal{S}_{\underline{\lambda}^{+}}$are contained in $\mathcal{H}_{l_{k+1}} \otimes \mathcal{S}_{l_{1}, \ldots, l_{k}}$ as a submodule with multiplicity 1 .

Because the proof for this theorem is again rather technical, it is postponed until the last section. Let us now consider a function $f \in \mathcal{C}^{\infty}\left(\mathbb{R}^{m}, \mathcal{S}_{\lambda^{+}}\right)$. When applying the twisted HSD-operator $\mathcal{Q}_{\lambda}^{T}$ on this function, we get that

$$
\mathcal{Q}_{\underline{\lambda}}^{T} f=\left(\prod_{i=1}^{k}\left(1+\frac{\underline{u}_{i} \underline{\partial}_{i}}{m+2 l_{i}-2 i}\right)\right) \underline{\partial}_{x} f
$$


is an element of $\operatorname{ker}\left(\underline{\partial}_{1}, \ldots, \underline{\partial}_{k},\left\langle\underline{u}_{1}, \underline{\partial}_{2}\right\rangle, \ldots,\left\langle\underline{u}_{k-1}, \underline{\partial}_{k}\right\rangle\right)$. However, it does no longer belong to $\operatorname{ker}\left(\underline{\partial}_{k+1},\left\langle\underline{u}_{k}, \underline{\partial}_{k+1}\right\rangle\right)$. Denoting the Laplace operator in $\underline{u}_{k+1}$ by means of $\Delta_{k+1}$, one can easily prove that

$$
\mathcal{Q}_{\underline{\lambda}}^{T} f \in \operatorname{ker}\left(\Delta_{k+1},\left\langle\underline{u}_{k}, \underline{\partial}_{k+1}\right\rangle^{2}\right),
$$

since $\Delta_{k+1}$ commutes with $\mathcal{Q}_{\underline{\lambda}}^{T}$, and

$$
\left\langle\underline{u}_{k}, \underline{\partial}_{k+1}\right\rangle \mathcal{Q}_{\underline{\lambda}}^{T} f=-\prod_{i=1}^{k-1}\left(1+\frac{\underline{u}_{i} \underline{\partial}_{i}}{m+2 l_{i}-2 i}\right) \frac{\underline{u}_{k} \underline{\partial}_{k+1}}{m+2 l_{k}-2 k} \underline{\partial}_{x} f .
$$

This implies that $\mathcal{Q}_{\underline{\lambda}}^{T} f$ takes values in $\mathcal{H}_{l_{k+1}} \otimes \mathcal{S}_{\underline{\lambda}}$ and explains our choice for $\mathbb{V}$ in (6). In order to derive the explicit expression for the HSD-operator $\mathcal{Q}_{\underline{\lambda}^{+}}$, we thus need the projection of $\mathcal{Q}_{\underline{\lambda}}^{T} f$ on $\mathcal{C}^{\infty}\left(\mathbb{R}^{m}, \mathcal{S}_{\underline{\lambda}^{+}}\right)$. Since we know that $\mathcal{Q}_{\underline{\lambda}}^{T} f$ is harmonic in $\underline{u}_{k+1}$, we can use the monogenic Fischer decomposition $(3)$ in this variable:

$$
\mathcal{Q}_{\underline{\lambda}}^{T} f=F_{l_{k+1}}+\underline{u}_{k+1} F_{l_{k+1}-1},
$$

where both functions $F_{j}$ are monogenic of degree $j$ in the variable $\underline{u}_{k+1}$. Applying $\underline{\partial}_{k+1}$ on both sides of this equation then yields

$$
F_{l_{k+1}-1}=-\frac{\underline{\partial}_{k+1} \mathcal{Q}_{\underline{\lambda}}^{T} f}{m+2 l_{k+1}+2} .
$$

Further calculations on the right-hand side of the latter expression lead to

$$
\begin{aligned}
F_{l_{k+1}-1} & =-\frac{1}{m+2 l_{k+1}+2} \prod_{i=1}^{k} \frac{m+2 l_{i}-2(i-1)}{m+2 l_{i}-2 i} \underline{\partial}_{k+1} \underline{\partial}_{x} f \\
& =\frac{2}{m+2 l_{k+1}+2} \prod_{i=1}^{k} \frac{m+2 l_{i}-2(i-1)}{m+2 l_{i}-2 i}\left\langle\underline{\partial}_{k+1}, \underline{\partial}_{x}\right\rangle f .
\end{aligned}
$$

For the operator appearing in the last equation, we have the following auxiliary result.

Lemma 2. The operator $\left\langle\underline{\partial}_{k+1}, \underline{\partial}_{x}\right\rangle$ is (up to a multiplicative constant) equal to the twistor operator defined by means of

$$
\mathcal{T}_{\underline{\lambda}^{-}}^{\underline{\lambda}^{+}}: \mathcal{C}^{\infty}\left(\mathbb{R}^{m}, \mathcal{S}_{\underline{\lambda}^{+}}\right) \rightarrow \mathcal{C}^{\infty}\left(\mathbb{R}^{m}, \mathcal{S}_{\underline{\lambda}^{-}}\right)
$$

Next, let us define $\pi_{\underline{\lambda}}\left[\underline{u}_{k+1}\right]$ as

$$
\pi_{\underline{\lambda}}\left[\underline{u}_{k+1}\right]:=\prod_{i=1}^{k}\left(1+\frac{\underline{u}_{i} \underline{\partial}_{i}}{m+2 l_{i}-2 i}\right) \underline{u}_{k+1} .
$$

In other words: this is the simplicial monogenic projection of the multiplication operator $\underline{u}_{k+1}$, defined by means of

$$
\pi_{\underline{\lambda}}\left[\underline{u}_{k+1}\right]: \mathcal{C}^{\infty}\left(\mathbb{R}^{m}, \mathcal{S}_{\underline{\lambda}^{-}}\right) \rightarrow \mathcal{C}^{\infty}\left(\mathbb{R}^{m}, \mathcal{H}_{l_{k+1}} \otimes \mathcal{S}_{\underline{\lambda}}\right) .
$$

One can then prove the following generalization of Proposition 1. 
Proposition 2. For all integers $l_{1} \geq l_{2} \geq \ldots \geq l_{k+1}>0$ and for all $f \in$ $\mathcal{C}^{\infty}\left(\mathbb{R}^{m}, \mathcal{S}_{\underline{\lambda}^{+}}\right)$with $\left\langle\underline{\partial}_{k+1}, \underline{\partial}_{x}\right\rangle f \neq 0$, there exists a unique constant $\gamma_{l_{k+1}} \in \mathbb{R}$ such that

$$
\mathcal{Q}_{\underline{\lambda}}^{T} f=\phi_{0}+\gamma_{l_{k+1}} \pi_{\underline{\lambda}}\left[\underline{u}_{k+1}\right]\left\langle\underline{\partial}_{k+1}, \underline{\partial}_{x}\right\rangle f,
$$

with $\phi_{0} \in \mathcal{C}^{\infty}\left(\mathbb{R}^{m}, \mathcal{S}_{\lambda^{+}}\right)$. This clearly means that $\phi_{0}=\mathcal{Q}_{\lambda^{+}} f$. The constant is given by $\gamma_{l_{k+1}}:=\frac{2}{2 l_{k+1}+m-2(k+1)}$.

Proof. Define a function $\phi_{0}$ by means of

$$
\phi_{0}:=\underline{\mathcal{Q}}_{\underline{\lambda}}^{T} f-\gamma_{l_{k+1}} \pi_{\underline{\lambda}}\left[\underline{u}_{k+1}\right]\left\langle\underline{\partial}_{k+1}, \underline{\partial}_{x}\right\rangle f,
$$

where the constant $\gamma_{l_{k+1}}$ is to fixed in such a way that $\phi_{0}$ meets the requirements of the proposition. The Fischer decomposition for $\mathcal{S}_{\underline{\lambda}}$-valued harmonic polynomials gives us that

$$
\mathcal{Q}_{\underline{\lambda}}^{T} f=F_{l_{k+1}}+\underline{u}_{k+1} F_{l_{k+1}-1},
$$

since $\mathcal{Q}_{\underline{\lambda}}^{T} f \in \operatorname{ker} \Delta_{k+1}$. Applying the Dirac operator $\underline{\partial}_{k+1}$ on both sides of the equality gives us that

$$
\begin{aligned}
& -\left(2 l_{k+1}+m-2\right) F_{l_{k+1}-1}= \\
& \quad \underline{\partial}_{k+1} \phi_{0}-\gamma_{l_{k+1}} \prod_{i=1}^{k}\left(\frac{2 l_{i}+m-2(i-1)}{2 l_{i}+m-2 i}\right)\left(m+2 l_{k+1}-2 k\right)\left\langle\underline{\partial}_{k+1}, \underline{\partial}_{x}\right\rangle f .
\end{aligned}
$$

Using (7), we then get that

$\underline{\partial}_{k+1} \phi_{0}=$

$$
\left(\gamma_{l_{k+1}}\left(2 l_{k+1}+m-2(k+1)\right)-2\right) \prod_{i=1}^{k}\left(\frac{2 l_{i}+m-2(i-1)}{2 l_{i}+m-2 i}\right)\left\langle\underline{\partial}_{k+1}, \underline{\partial}_{x}\right\rangle f .
$$

If we choose

$$
\gamma_{l_{k+1}}=\frac{2}{2 l_{k+1}+m-2(k+1)}
$$

one can directly verify that the proposition holds.

Schematically, we can represent this decomposition as follows:

$$
\begin{aligned}
& \mathcal{C}^{\infty}\left(\mathbb{R}^{m}, \mathcal{S}_{\underline{\lambda}^{+}}\right)-\mathcal{Q}_{k}^{T}>\mathcal{C}^{\infty}\left(\mathbb{R}^{m}, \mathcal{H}_{l_{k+1}} \otimes \mathcal{S}_{\underline{\lambda}}\right)
\end{aligned}
$$

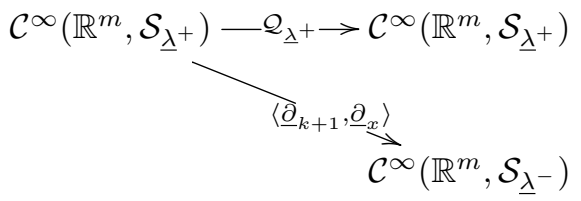

Remark 6. In case $f \in \mathcal{C}^{\infty}\left(\mathbb{R}^{m}, \mathcal{S}_{\lambda^{+}}\right) \cap \operatorname{ker}\left\langle\underline{\partial}_{k+1}, \underline{\partial}_{x}\right\rangle$, the proposition above immediately reduces to

$$
\mathcal{Q}_{\underline{\lambda}}^{T} f \in \mathcal{C}^{\infty}\left(\mathbb{R}^{m}, \mathcal{S}_{\underline{\lambda}^{+}}\right)
$$




\section{Technical proofs}

In this section, we have gathered the proofs for technical results which were omitted in the preceeding text.

\subsection{Representations of order 2}

Let us first take a look at the tensor product $\mathcal{H}_{l_{1}} \otimes \mathcal{H}_{l_{2}}$. It was proven in [16] that

Lemma 3. For all integers $l_{1} \geq l_{2}>0$, we have that

$$
\left(l_{1}, 0, \cdots, 0\right) \otimes\left(l_{2}, 0, \cdots, 0\right) \cong \bigoplus_{i=0}^{l_{2}} \bigoplus_{j=0}^{i}\left(l_{1}+l_{2}-2 i+j, j, 0, \cdots, 0\right),
$$

where each highest weight refers to an irreducible representation for the spin group.

We can nicely represent this in the following scheme:

$$
\begin{aligned}
& \begin{array}{llllllll}
\left(l_{1}, l_{2}\right) & \oplus & \left(l_{1}-1, l_{2}-1\right) & \oplus & \cdots & \oplus & \left(l_{1}-l_{2}+1,1\right) & \oplus \quad\left(l_{1}-l_{2}, 0\right)
\end{array} \\
& \begin{array}{lllll}
\left(l_{1}+l_{2}-j, j\right) & \oplus & \cdots & \left(l_{1}+l_{2}-2 j, 0\right)
\end{array} \\
& \left(l_{1}+l_{2}-1,1\right) \quad \underset{\oplus}{\dot{\oplus}}\left(l_{1}+l_{2}, 0\right) \quad\left(l_{1}+l_{2}-2,0\right)
\end{aligned}
$$

In this decomposition, each summand appears with multiplicity 1. Using this lemma, Theorem 1 from Section 4 can now be proven.

Theorem 1. For each pair of integers $l_{1} \geq l_{2}>0$, we have that $\mathcal{S}_{l_{1}, l_{2}}$ and $\mathcal{S}_{l_{1}, l_{2}-1}$ are $\operatorname{Spin}(m)$-submodules of $\mathcal{H}_{l_{2}} \otimes \mathcal{S}_{l_{1}}$ with multiplicity 1.

Proof. In view of our polynomial models, it is easily seen that $\mathcal{S}_{l_{1}, l_{2}}$ is a subset of $\mathcal{H}_{l_{2}} \otimes \mathcal{S}_{l_{1}}$. It is multiplicity-free, since

$$
\mathcal{H}_{l_{2}} \otimes \mathcal{S}_{l_{1}} \subset \mathcal{H}_{l_{1}} \otimes \mathcal{H}_{l_{2}} \otimes \mathbb{S}
$$

and $\mathcal{S}_{l_{1}, l_{2}}$ only appears as a submodule of $\mathcal{H}_{l_{1}, l_{2}} \otimes \mathbb{S}$, using (8) and (1). The vector space $\mathcal{S}_{l_{1}, l_{2}-1}$ however, is a submodule of $\mathcal{H}_{l_{1}} \otimes \mathcal{H}_{l_{2}} \otimes \mathbb{S}$ with multiplicity 2 , since it is both a submodule of $\mathcal{H}_{l_{1}, l_{2}} \otimes \mathbb{S}$ and $\mathcal{H}_{l_{1}+1, l_{2}-1} \otimes \mathbb{S}$, again using (8) and (1). On the other hand, we also have that

$$
\mathcal{H}_{l_{1}} \otimes \mathcal{H}_{l_{2}} \otimes \mathbb{S} \cong\left(\mathcal{H}_{l_{2}} \otimes \mathcal{S}_{l_{1}}\right) \oplus\left(\mathcal{H}_{l_{2}} \otimes \mathcal{S}_{l_{1}-1}\right) .
$$

If one can prove that $\mathcal{H}_{l_{2}} \otimes \mathcal{S}_{l_{1}-1}$ has $\mathcal{S}_{l_{1}, l_{2}-1}$ as a submodule with multiplicity 1 , then the theorem is proven. This indeed is the case since

$$
\mathcal{H}_{l_{1}-1} \otimes \mathcal{H}_{l_{2}} \otimes \mathbb{S} \cong\left(\mathcal{H}_{l_{2}} \otimes \mathcal{S}_{l_{1}-1}\right) \oplus\left(\mathcal{H}_{l_{2}} \otimes \mathcal{S}_{l_{1}-2}\right),
$$

and the module $\mathcal{S}_{l_{1}, l_{2}-1}$ is contained in the subrepresentation $\mathcal{H}_{l_{1}, l_{2}-1} \otimes \mathbb{S}$ of the tensor product $\left(\mathcal{H}_{l_{1}-1} \otimes \mathcal{H}_{l_{2}}\right) \otimes \mathbb{S}$ with multiplicity 1 . It is however not a submodule of $\mathcal{H}_{l_{2}} \otimes \mathcal{S}_{l_{1}-2}$, since

$$
\mathcal{H}_{l_{2}} \otimes \mathcal{S}_{l_{1}-2} \subset \mathcal{H}_{l_{1}-2} \otimes \mathcal{H}_{l_{2}} \otimes \mathbb{S}
$$


and $\mathcal{S}_{l_{1}, l_{2}-1}$ is not a submodule of $\mathcal{H}_{l_{1}-2} \otimes \mathcal{H}_{l_{2}} \otimes \mathbb{S}$, again because of (8) and (1).

\subsection{Representations of order $k$}

Let $\mathbb{V}$ be an arbitrary representation of $\operatorname{Spin}(m)$, or its Lie algebra $\mathfrak{s o}(m)$. Denote by $\Gamma_{\lambda}$ the finite-dimensional irreducible representation with highest weight $\lambda$. The multiplicity of $\Gamma_{\lambda}$ in $\mathbb{V}$ is denoted by $m_{\lambda}(\mathbb{V})$, and the multiplicity of an arbitrary weight $\mu$ in $\Gamma_{\lambda}$ is denoted by $n_{\mu}\left(\Gamma_{\lambda}\right)$. We will use the following result (see e.g. [15]).

Theorem 3. If $\nu$ is a dominant weight such that $m_{\nu}\left(\Gamma_{\lambda} \otimes \Gamma_{\mu}\right)>0$, then there is a weight $\mu^{\prime}$ of $\Gamma_{\mu}$ such that $\nu=\lambda+\mu^{\prime}$. Moreover, if this is the case, then we at the same time have that $m_{\nu}\left(\Gamma_{\lambda} \otimes \Gamma_{\mu}\right) \leq n_{\lambda-\nu}\left(\Gamma_{\mu}\right)$.

Put $\underline{\lambda}^{-}=\left(l_{1}, l_{2}, \ldots, l_{k}, l_{k+1}-1\right)$ and $\underline{\lambda}^{+}=\left(l_{1}, l_{2}, \ldots, l_{k}, l_{k+1}\right)$, as above. In view of definitions 1 and 2 , it is easily seen that $\mathcal{S}_{\lambda^{+}}$indeed is contained as a submodule in the tensor product $\mathcal{H}_{l_{k+1}} \otimes \mathcal{S}_{\lambda}$, since all polynomials in $\mathcal{S}_{\underline{\lambda}^{+}}$indeed are simplicial monogenic in the first $k$ variables and harmonic in $\underline{u}_{k+1}$. Recalling the definition of the projection operator $\pi_{\underline{\lambda}}\left[\underline{u}_{k+1}\right]$, it is also clear that

$$
\prod_{i=1}^{k}\left(1+\frac{\underline{u}_{i} \underline{\partial}_{i}}{m+2 l_{i}-2 i}\right)\left[\underline{u}_{k+1}\right] \mathcal{S}_{\underline{\lambda}^{-}} \subset \mathcal{H}_{l_{k+1}} \otimes \mathcal{S}_{l_{1}, \ldots, l_{k}}
$$

whence $\mathcal{S}_{\underline{\lambda}^{-}}$is, up to an embedding factor, also contained in $\mathcal{H}_{l_{k+1}} \otimes \mathcal{S}_{l_{1}, \ldots, l_{k}}$ We then proceed with the proof of Theorem 2 to obtain uniqueness (in two parts).

Theorem 2 (I). The vector space $\mathcal{S}_{\underline{\lambda}^{-}}$is contained as a submodule inside the tensor product $\mathcal{H}_{l_{k+1}} \otimes \mathcal{S}_{l_{1}, \ldots, l_{k}}$ with multiplicity 1.

We already have shown that $m_{\underline{\lambda}^{-}}\left(\mathcal{H}_{l_{k+1}} \otimes \mathcal{S}_{l_{1}, \ldots, l_{k}}\right)>0$. Putting $\lambda=\left(l_{1}, \ldots, l_{k}\right)^{\prime}$, $\mu=\left(l_{k+1}\right)$ and $\nu=\left(\underline{\lambda}^{-}\right)^{\prime}$ in Theorem 3, we then need to prove that $n_{\lambda-\nu}\left(\Gamma_{\mu}\right)=1$, which will lead to the first part of Theorem 2 . We see that $\lambda-\nu=\left(0, \ldots, 0,-l_{k+1}+1,0, \ldots, 0\right)$, where the nonzero element is on the $(k+1)$-th position. Due to the action of the Weyl-group, we know that

$$
n_{\left(0, \ldots, 0,-l_{k+1}+1,0, \ldots, 0\right)}\left(\mathcal{H}_{l_{k+1}}\right)=n_{\left(l_{k+1}-1\right)}\left(\mathcal{H}_{l_{k+1}}\right) .
$$

In order to calculate the multiplicity of the weight $\left(l_{k+1}-1\right)$ in the $\operatorname{Spin}(m)$ representation $\mathcal{H}_{l_{k+1}}$, we make use of Freudenthal's formula, which we state in the following theorem (in a form adapted to our needs).

Theorem 4. Let $\Gamma_{\lambda}$ be an irreducible representation with highest weight $\lambda$ for $\mathfrak{g}=\mathfrak{s o}(m)$. The multiplicity $n_{\mu}\left(\Gamma_{\lambda}\right)$ of the weight $\mu$ in $\Gamma_{\lambda}$ is given recursively, by means of

$$
\left(2\langle\lambda-\mu, \mu+\delta\rangle+\|\lambda-\mu\|^{2}\right) n_{\mu}\left(\Gamma_{\lambda}\right)=2 \sum_{\alpha \in \Delta^{+}} \sum_{a \geq 1}\langle\mu+\alpha a, \alpha\rangle n_{\mu+\alpha a}\left(\Gamma_{\lambda}\right)
$$


Hereby, $\delta$ stands for half the sum of the positive roots, $\langle\cdot, \cdot\rangle$ is the Killing form and $\Delta^{+}$is the set of positive roots.

In our case, i.e. for the Lie algebra $\mathfrak{s o}(m)=\mathfrak{s o}(2 n+1)$, we get

$$
\begin{aligned}
\Delta^{+} & =\{(1,0, \ldots, 0),(0,1,0, \ldots, 0), \ldots,(0, \ldots, 0,1), \\
& (1,1,0, \ldots, 0),(1,0,1,0, \ldots, 0), \ldots,(0, \ldots, 0,1,1), \\
& (1,-1,0, \ldots, 0),(1,0,-1,0, \ldots, 0), \ldots,(0, \ldots, 0,1,-1)\} .
\end{aligned}
$$

and the Killing form is the standard inner product. Thus $\delta=\left(n-\frac{1}{2}, n-\right.$ $\left.\frac{3}{2}, \ldots, \frac{1}{2}\right)$, with $m=2 n+1$. Putting $\lambda=\left(l_{k+1}, 0, \ldots, 0\right)$ and $\mu=\left(l_{k+1}-\right.$ $1,0, \ldots, 0)$, the left-hand side of the Freudenthal formula becomes $2\left(l_{k+1}+\right.$ $n-1) n_{\mu}\left(\Gamma_{\lambda}\right)$. In order to determine the right-hand side of the equation, it suffice to note that the only non-trivial $\mu+\alpha a$ that will appear in the sum are of the form

$$
\left(l_{k+1}, 0, \ldots, 0\right),\left(l_{k+1}-1,1,0, \ldots, 0\right), \ldots,\left(l_{k+1}-1,0, \ldots, 0,1\right),
$$

where $\alpha$ is $(1,0, \ldots, 0),(0,1,0, \ldots, 0), \ldots,(0,0, \ldots, 0,1)$ respectively, and $a=$ 1. There are no other possibilities, in view of the following classical result.

Theorem 5. If $\lambda=\left(\lambda_{1}, \ldots, \lambda_{k}\right)$ is the highest weight of an irreducible representation $\Gamma_{\lambda}$, and $\mu=\left(\mu_{1}, \ldots, \mu_{k}\right)$ is a non-trivial weight in $\Gamma_{\lambda}$, then

$$
\sum_{i=1}^{k}\left|\mu_{i}\right| \leq \sum_{i=1}^{k}\left|\lambda_{i}\right| .
$$

We then get that

$$
\begin{aligned}
2 & \sum_{\alpha \in \Delta^{+}} \sum_{a \geq 1}\langle\mu+\alpha a, \alpha\rangle n_{\mu+\alpha a}\left(\Gamma_{\lambda}\right) \\
& =2 l_{k+1} n_{\left(l_{k+1}\right)}\left(\Gamma_{\left(l_{k+1}\right)}\right)+2 \sum_{i=2}^{n} n_{\left(l_{k+1}-1\right)+\delta_{i}}\left(\Gamma_{l_{k+1}}\right),
\end{aligned}
$$

where $\delta_{i}=(0, \ldots, 0,1,0, \ldots, 0)$, with 1 on the $i$-th position. If one can now prove that for each $2 \leq i \leq n$ one also has that $n_{\left(l_{k+1}-1\right)+\delta_{i}}\left(\Gamma_{l_{k+1}}\right)=1$, our proof is complete. This indeed is the case, as follows from Freudenthal's formula again. Putting $\lambda=\left(l_{k+1}\right)$ and $\mu=\left(l_{k+1}-1\right)+\delta_{i}$, the left-hand side of the formula becomes $2\left(l_{k+1}+i-2\right) n_{\left(l_{k+1}-1\right)+\delta_{i}}\left(\Gamma_{\left(l_{k+1}\right)}\right)$. For the right-hand side, the only possible values for $\mu+\alpha a$ in the sum are $\left(l_{k+1}\right),\left(l_{k+1}-1\right)+$ $\delta_{1}, \ldots,\left(l_{k+1}-1\right)+\delta_{i-1}$. Induction on $i$ indeed gives us that

$$
n_{\left(l_{k+1}-1\right)+\delta_{i}}\left(\Gamma_{\left(l_{k+1}\right)}\right)=1,
$$

for each $2 \leq i \leq n$.

Finally, we also prove the remaining part of Theorem 2 .

Theorem 2 (II). The vector space $\mathcal{S}_{\underline{\lambda}^{+}}$is contained as a submodule inside the tensor product $\mathcal{H}_{l_{k+1}} \otimes \mathcal{S}_{l_{1}, \ldots, l_{k}}$ with multiplicity 1. 
Proof. We once again make use of Theorem 3. Putting $\lambda=\left(l_{1}, \ldots, l_{k}\right)^{\prime}, \mu=$ $\left(l_{k+1}\right)$ and $\nu=\left(\underline{\lambda}^{+}\right)^{\prime}$, this theorem states that

$$
m_{\left(\underline{\lambda}^{+}\right)^{\prime}}\left(\Gamma_{\left(l_{1}, \ldots, l_{k}\right)^{\prime}} \otimes \Gamma_{\left(l_{k+1}\right)}\right) \leq n_{\left(0, \ldots, 0,-l_{k+1}, 0, \ldots, 0\right)}\left(\Gamma_{\left(l_{k+1}\right)}\right)
$$

Due to the action of the Weyl group,

$$
n_{\left(0, \ldots, 0,-l_{k+1}, 0, \ldots, 0\right)}\left(\Gamma_{\left(l_{k+1}\right)}\right)=n_{\left(l_{k+1}\right)}\left(\Gamma_{\left(l_{k+1}\right)}\right)=1,
$$

as this is the multiplicity of the highest weight space.

\section{Conclusion}

In this article, we have shown that we can always deduce the higher spin Dirac operator in $(k+1)$ variables from the HSD-operator in 1 variable less, using a procedure which we have called 'twisting higher spin Dirac operators'. This will prove to be an invaluable step towards determining the set of (polynomial) null solutions of arbitrary higher spin Dirac operators, as we will elaborate in future work.

\section{References}

[1] Brackx, F., Delanghe, R., Sommen, F., Clifford Analysis, Research Notes in Mathematics 76, Pitman, London, 1982.

[2] Brackx, F., Eelbode, D., Van de Voorde, L., Polynomial solutions for higher spin Dirac operators in three vector variables, submitted.

[3] Branson, T., Stein-Weiss operators and ellipticity, J. Funct. Anal. 151 No. 2 (1997), pp. 334-383.

[4] Bureš, J., Sommen, F., Souček, V., Van Lancker, P., Rarita-Schwinger type operators in Clifford analysis, Journal of Funct. Anal. 185, pp. 425-456.

[5] Bureš, J., Sommen, F., Souček, V., Van Lancker, P., Symmetric analogues of Rarita-Schwinger equations, Ann. Glob. Anal. Geom. 21 No. 3 (2001), pp. 215240.

[6] Constales, D., Sommen. F., Van Lancker, P., Models for irreducible representations of Spin $(m)$, Adv. Appl. Clifford Algebras 11 No. S1 (2001), pp. 271-289.

[7] De Bie, H., Harmonic and Clifford analysis in superspace, Ph.D. thesis, Ghent University (2008).

[8] Delanghe, R., Sommen, F., Souček, V., Clifford analysis and spinor valued functions, Kluwer Academic Publishers, Dordrecht, 1992.

[9] De Schepper, H., Eelbode, D., Raeymaekers, T., On a special type of solutions for arbitrary higher spin Dirac Operators, J. Phys. A: Math. Theor. 43 (2010) 325208 (13pp).

[10] Eelbode, D., Šmíd, D., Factorization of Laplace operators on higher spin representations, Complex Analysis and Operator Theory 6(5) (2012), pp. 1011-1023.

[11] Eelbode, D., Van de Voorde, L., A toy model for higher spin Dirac operators, Physics of Atomic Nuclei 73(2) (2010) pp. 282-287.

[12] Fegan, H. D., Conformally invariant first order differential operators, Quart. J. Math. 27 (1976), pp. 513-538. 
[13] Gilbert, J., Murray, M.A.M., Clifford algebras and Dirac operators in harmonic analysis, Cambridge University Press, Cambridge, 1991.

[14] Howe, R., Tan, E., Willenbring, J., Stable branching rules for classical symmetric pairs, Trans. of the AMS, 357(4) (2004), pp. 16011626.

[15] Humphreys, J., Introduction to Lie algebra and representation theory, SpringerVerlag, New York, 1972.

[16] Klimyk, A. U., Infinitesimal operators for representations of complex Lie groups and Clebsch-Gordan coefficients for compact groups, J. Phys. A: Math. Gen 15 (1982), pp. 3009-3023.

[17] Slovak, J., Natural operators on conformal manifolds, Masaryk University Dissertation (Brno, 1993)

[18] Stein, E.W. , Weiss, G., Generalization of the Cauchy-Riemann equations and representations of the rotation group, Amer. J. Math. 90 (1968), pp. 163-196.

H. De Schepper

Clifford research group, Department of Mathematical Analysis, Ghent University, Galglaan 2, 9000 Ghent, Belgium

e-mail: hds@cage.ugent.be

D. Eelbode

Department of Mathematics and Computer Science, University of Antwerp, Campus Middelheim, G-Building, Middelheimlaan 1, 2020 Antwerpen, Belgium

e-mail: david.eelbode@ua.ac.be

T. Raeymaekers

Clifford research group, Department of Mathematical Analysis, Ghent University, Galglaan 2, 9000 Ghent, Belgium

e-mail: tr@cage.ugent.be 